\title{
Cost Allocation in Shortest Path Games
}

\author{
Mark Voorneveld ${ }^{1}$ \\ Department of Mathematics, University of Utrecht, P.O.Box 80010, 3508 TA Utrecht, \\ The Netherlands. M.Voorneveld@math.uu.nl
}

Sofia Grahn ${ }^{2}$

Department of Economics, Uppsala University, P.O.Box 513, 75120 Uppsala, Sweden. Sofia.Grahn@nek.uu.se

\begin{abstract}
A class of cooperative games arising from shortest path problems is defined. These shortest path games are shown to be totally balanced and allow a populationmonotonic allocation scheme. Possible methods for obtaining core elements are indicated; first, by relating to the allocation rules in taxation and bankruptcy problems, second, by constructing an explicit rule that takes opportunity costs into account by considering the costs of the second best alternative and that rewards players who are crucial to the construction of the shortest path. Finally, noncooperative games arising from shortest path problems are introduced, in which players make bids or claims on paths. The core allocations of the cooperative shortest path game coincide with the payoff vectors in the strong Nash equilibria of the associated noncooperative shortest path game.
\end{abstract}

Keywords: shortest path games, population-monotonic allocation schemes, core, strong Nash equilibrium.

JEL codes: C70, C72.

\footnotetext{
${ }^{1}$ Corresponding author.

${ }^{2}$ This author acknowledges the hospitality of the Department of Econometrics at Tilburg University during part of the research.
} 


\section{Introduction}

The operations research literature offers numerous algorithms and heuristics for network optimization problems. In game theoretic approaches to network optimization problems, different agents control the elements of the network. Next to finding optimal solutions, this adds the problem of dividing the costs or benefits generated by such solutions over the involved agents. Curiel (1997) provides an overview of several classes of games arising from problems in operations research.

Only recently, Fragnelli, García-Jurado, and Méndez-Naya (1999) have initiated a game theoretic approach to shortest path problems. They study a class of cooperative shortest path games in which the transportation of a good from a source to a sink of a network generates an externally given income. The nodes in the network are owned by the players. The value of a coalition is defined by the net income this coalition can realize by transporting the good through the network via a shortest path, using only nodes that are owned by the players in the coalition (or zero, if the net income happens to be negative). The corresponding class of cooperative games is shown to coincide with the class of monotonic games. Therefore, shortest path games need not have core elements. For a shortest path game to be balanced, two rather strong restrictions have to be fulfilled: a certain reduced game needs to be balanced, and certain veto players have to take important positions in the game. Their paper ends with an axiomatization of the Shapley value of shortest path games.

As opposed to the model of Fragnelli et al. (1999), where the income or reward for transporting a good from source to sink is not associated with a specific player, the present paper introduces a class of cooperative games arising from shortest path problems where there is a reward (possibly equal to zero) for each player if he transports his goods from source to sink. The definition of the cooperative shortest path games is given in Section 2 . In Section 3, it is shown that these shortest path games are (totally) balanced. Possible methods for obtaining core elements are indicated by relating to the allocation rules in taxation (cf. Young, 1987, 1990) and bankruptcy problems (cf. Aumann and Maschler, 1985). Every efficient allocation in which each player contributes a nonnegative amount not exceeding his reward to the costs of the shortest path, yields a core element of the shortest path game. Sprumont (1990) introduces the notion of a population-monotonic allocation scheme (PMAS) for a cooperative game $(N, v)$. A PMAS specifies for each coalition $S \subseteq N$ of players an allocation of the value $v(S)$ over its members. Moreover, it reflects the intuition that there is 'strength in numbers': for each $i \in N$ and each pair of coalitions $S, T$ with $i \in S \subseteq T \subseteq N$, the share of $v(T)$ allocated to $i$ is at least as large as the share of $v(S)$ allocated to $i$. It is shown in Section 3 that each shortest path game has a PMAS. In Section 4, an allocation rule is constructed that takes opportunity costs into account by considering the costs of the second best alternative and that rewards players who are crucial to the construction of the shortest path. Finally, Section 5 introduces noncooperative games arising from shortest path problems. In such a strategic game, players make bids or claims on certain paths. It is shown that the core allocations of the cooperative shortest path game exactly coincide with the payoff vectors in the strong 
Nash equilibria of the associated noncooperative shortest path game.

Notation: For a finite set $N, 2^{N}=\{S \mid S \subseteq N\}$ denotes the collection of all subsets of $N$. For $\left(x_{i}\right)_{i \in N}$, where $x_{i} \in \mathbb{R}$ for each $i \in N$, and for a subset $S \subseteq N$, we denote $x(S)=\sum_{i \in S} x_{i}$. $\mathbb{R}$ denotes the set of reals, $\mathbb{R}_{+}=[0, \infty)$ the set of nonnegative reals. For $a, b \in \mathbb{R}$ we define $a \wedge b:=\min \{a, b\}$. The end of proofs is indicates with the symbol $\square$, the end of definitions, examples, and remarks with the symbol $\triangleleft$.

\section{Shortest path games}

In this section shortest path problems and their associated cooperative games are defined. In the shortest path problems considered in this paper, there is a finite set of players. Each player owns arcs or connections in a finite network. There are costs associated to the use of each arc. Each player receives a nonnegative reward if he manages to transport a good from the source of the network to its sink.

Definition 2.1 A shortest path problem is a tuple $\left\langle N, V,\left(A_{i}\right)_{i \in N}, w,\left(r_{i}\right)_{i \in N}\right\rangle$, where

- $N$ is a finite set of players;

- $V$ is a finite set of vertices with two special elements: the source So and the sink $S i$

- each player $i \in N$ owns a set $A_{i} \subseteq V \times V$ of directed arcs in the network;

- the function $w: \cup_{i \in N}\{i\} \times A_{i} \rightarrow \mathbb{R}_{+}$assigns a weight (or length, or cost) to all the arcs owned by the players. The weight assigned to arc $(a, b)$ owned by player $i \in N$ is $w(i,(a, b)) \in \mathbb{R}_{+}$;

- each player $i \in N$ receives a reward $r_{i} \in \mathbb{R}_{+}$for transporting his goods from the source to the sink.

Notice that more than one player can own an arc between two vertices, and that the costs of an arc can depend on its owner. Several definitions and results in this paper are illustrated with the following simple example.

Example 2.2 The shortest path problem with player set $N=\{1,2,3\}$, vertex set $V=\{S o, S i, v\}$, the players respectively owning arc sets $A_{1}=\{(S o, v),(S o, S i)\}, A_{2}=$ $\{(v, S i)\}, A_{3}=\{(S o, S i)\}$, and weights $w(1,(S o, v))=w(2,(v, S i))=1, w(1,(S o, S i))=$ $3, w(3,(S o, S i))=6$ is depicted in Figure 1 (where it is assumed that arcs are directed from left to right). The numbers $2 ; 1$ next to the arc $(v, S i)$, for instance, indicate that this arc is owned by player 2 and that the costs of this arc owned by player 2 equal 1 . Take the rewards equal to $r_{1}=r_{2}=r_{3}=8$. 


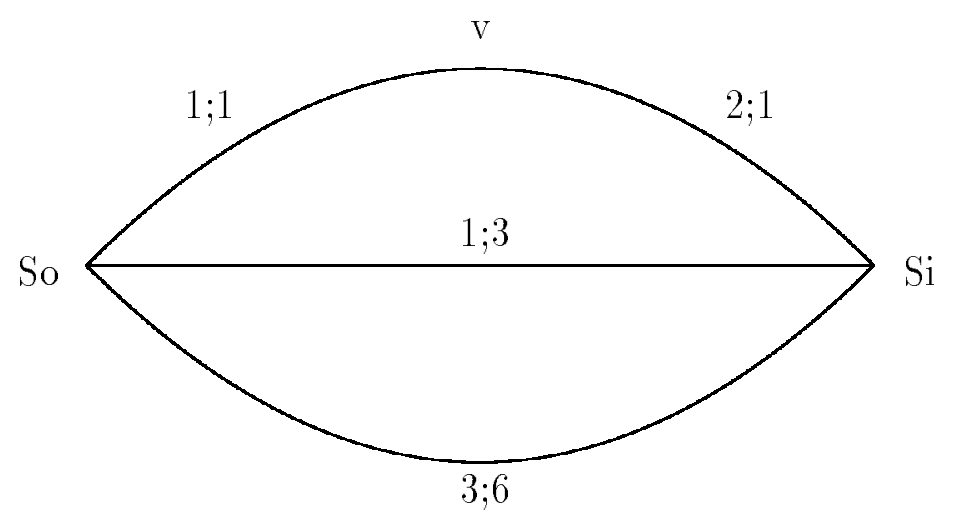

Figure 1: A shortest path problem

Let $S \in 2^{N} \backslash\{\emptyset\}$ be a coalition of players. A path owned by the players in $S$ is a sequence $\left(v_{1}, i_{1}, v_{2}, i_{2}, \ldots, i_{m-1}, v_{m}\right)$ of vertices $v_{k}$ and players $i_{k}$ such that $v_{1}=S o, v_{m}=S i$, and for each $k \in\{1, \ldots, m-1\}$ the arc $\left(v_{k}, v_{k+1}\right)$ is owned by player $i_{k} \in S$. Let $P(S)$ denote the collection of all paths owned by coalition $S$.

The costs associated to a path $p=\left(v_{1}, i_{1}, v_{2}, i_{2}, \ldots, i_{m-1}, v_{m}\right) \in P(S)$ are defined as the sum of the costs of its arcs:

$$
\operatorname{cost}(p)=\sum_{k=1}^{m-1} w\left(i_{k},\left(v_{k}, v_{k+1}\right)\right) .
$$

Obviously, if a coalition $S$ has to go from source to sink, it will choose among its alternatives in $P(S)$ the path with minimal costs. Define for each $S \in 2^{N} \backslash\{\emptyset\}$ :

$$
c(S)= \begin{cases}\min _{p \in P(S)} \operatorname{cost}(p) & \text { if } P(S) \neq \emptyset, \\ \infty & \text { otherwise. }\end{cases}
$$

Shortest paths in directed networks can be determined, for instance, by the algorithm of Dijkstra (1959).

From now on, when we refer to 'a shortest path' without explicitly stating a coalition owning it, we mean a shortest path owned by the grand coalition $N$.

Recall that a cooperative game with transferable utility is a tuple $(N, v)$, where $N$ is a finite set of players and $v: 2^{N} \backslash\{\emptyset\} \rightarrow \mathbb{R}$ is a function that assigns to each coalition $S \in 2^{N} \backslash\{\emptyset\}$ its value $v(S) \in \mathbb{R}$.

The cooperative game associated with a shortest path problem reflects the following intuition: if a coalition $S \in 2^{N} \backslash\{\emptyset\}$ transports its goods from source to sink, it will receive a total reward $r(S)=\sum_{i \in S} r_{i}$ and incur costs $c(S)$, the costs of the cheapest alternative $S$ has to go from source to sink. If $r(S)-c(S)>0$, coalition $S$ makes a profit. If $r(S)-c(S) \leq 0$, coalition $S$ can generate profit zero by simply doing nothing. Therefore, coalition $S$ can make a profit $\max \{r(S)-c(S), 0\}$. 
Definition 2.3 Let $\left\langle N, V,\left(A_{i}\right)_{i \in N}, w,\left(r_{i}\right)_{i \in N}\right\rangle$ be a shortest path problem. The associated shortest path game $(N, v)$ is defined as follows:

$$
\forall S \in 2^{N} \backslash\{\emptyset\} \quad: \quad v(S)=\max \{r(S)-c(S), 0\} .
$$

Let $S, T \in 2^{N} \backslash\{\emptyset\}, S \subseteq T$. Then $r(S) \leq r(T)$ and $c(S) \geq c(T)$, so $v(S) \leq v(T)$ : the shortest path game $(N, v)$ is monotonic.

To avoid trivialities, assume that

$$
0<c(N)<r(N)
$$

This assumption implies that

- there is a player $i \in N$ with a strictly positive reward $r_{i}$;

- the value $v(N)$ of the grand coalition is positive;

- the shortest path has positive length $c(N)>0$.

This avoids the zero game and makes sure that there are indeed costs to divide over the players.

Example 2.4 In the shortest path problem of Example 2.2, coalition $\{2\}$ is the only coalition not owning a path: $c(\{2\})=\infty$. The cooperative game associated with the shortest path problem is given by $v(\{1\})=\max \left\{r_{1}-c(\{1\}), 0\right\}=\max \{8-3,0\}=5, v(\{2\})=$ $\max \left\{r_{2}-c(\{2\}), 0\right\}=\max \{8-\infty, 0\}=0, v(\{3\})=2, v(\{1,2\})=14, v(\{1,3\})=$ $13, v(\{2,3\})=10, v(N)=22$.

Recall that the core of a game $(N, v)$ is the set

$$
C(N, v)=\left\{x \in \mathbb{R}^{N} \mid x(N)=v(N) \text { and } x(S) \geq v(S) \text { for each } S \in 2^{N} \backslash\{\emptyset\}\right\} .
$$

In the next section, the core of shortest path games is shown to be nonempty.

\section{Core allocations of shortest path games}

Fragnelli et al. (1999) indicate that in a class of shortest path games where there is a single reward, provided by someone outside the player set, the core may be empty. The purpose of the present section is to indicate that every shortest path game as formulated in Definition 2.3 is totally balanced and has a population-monotonic allocation scheme. Every efficient allocation in which each player contributes a nonnegative amount not exceeding his reward to the costs of the shortest path, yields a core element. 
Proposition 3.1 Let $\left\langle N, V,\left(A_{i}\right)_{i \in N}, w,\left(r_{i}\right)_{i \in N}\right\rangle$ be a shortest path problem and $(N, v)$ the associated shortest path game. Take

$$
B=\left\{x \in \mathbb{R}^{N} \mid x(N)=v(N) \text { and } x_{i} \in\left[0, r_{i}\right] \text { for each } i \in N\right\} .
$$

Then $B \subseteq C(N, v)$.

Proof. Let $x \in B$. Then $x(N)=v(N)$ by definition, so it remains to show that $x(S) \geq v(S)$ for each $S \in 2^{N} \backslash\{\emptyset\}$. Let $S \in 2^{N} \backslash\{\emptyset\}$. If $v(S)=0$, then $x(S) \geq 0=v(S)$, since $x_{i} \geq 0$ for each player $i \in N$. So assume that $v(S)=r(S)-c(S)>0$.

Define for each $i \in N$ the number $\mu_{i}=\frac{r_{i}-x_{i}}{c(N)}$ to be the share of the costs $c(N)$ of the shortest path paid by player $i \in N$. By assumption (1), $\mu_{i}$ is well-defined. By definition of $B, \mu_{i} \in\left[0, \frac{r_{i}}{c(N)}\right]$ and $\sum_{i \in N} \mu_{i}=1$. Moreover, $x_{i}=r_{i}-\mu_{i} c(N)$. Consequently,

$$
\begin{aligned}
x(S) & =\sum_{i \in S}\left(r_{i}-\mu_{i} c(N)\right) \\
& =r(S)-\left(\sum_{i \in S} \mu_{i}\right) c(N) \\
& \geq r(S)-1 * c(S) \\
& =v(S) .
\end{aligned}
$$

The weak inequality follows from $\sum_{i \in S} \mu_{i} \leq 1$ and $c(N) \leq c(S)$. This proves that $x \in C(N, v)$.

To prove that a shortest path game is balanced, it suffices to show that the set $B$ in Proposition 3.1 is nonempty. This follows from assumption (1). Assume for instance, that each player contributes a share of the costs that is proportional to his reward:

$$
\forall i \in N \quad: \quad x_{i}=r_{i}-\frac{r_{i}}{r(N)} c(N)
$$

Then clearly $x(N)=r(N)-c(N)=v(N)$ and assumption (1) implies that

$$
x_{i}=r_{i}\left(1-\frac{c(N)}{r(N)}\right) \in\left[0, r_{i}\right] .
$$

Hence, this proportional allocation of the costs yields a core allocation. For each coalition $S \in 2^{N} \backslash\{\emptyset\}$, the subgame $(S, v)$ of the shortest path game $(N, v)$ is again a shortest path game and therefore balanced. Consequently, every shortest path game is totally balanced.

Sprumont (1990) introduces the notion of a population-monotonic allocation scheme (PMAS) for a cooperative game $(N, v)$. A PMAS specifies for each coalition $S \subseteq N$ of players an allocation of $v(S)$ over its members. Moreover, it reflects the intuition that there is 'strength in numbers': the share allocated to each player $i \in N$ is nondecreasing 
in the size of the coalitions of which he is a member. Formally, a PMAS for the game $(N, v)$ is a vector $\left(x_{S, i}\right)_{S \subseteq N, i \in S}$ of real numbers such that

$$
\forall S \subseteq N: \quad \sum_{i \in S} x_{S, i}=v(S)
$$

and

$$
\forall S, T \subseteq N, \forall i \in S: \text { if } S \subseteq T \text {, then } x_{S, i} \leq x_{T, i} .
$$

Not all cooperative games have a PMAS. A necessary, but not sufficient, condition is that the game $(N, v)$ is totally balanced; see Sprumont (1990, p.380). Define $x_{S, i}$ in such a way that player $i$ pays a proportional share of $c(S)$ (or zero if $v(S)=0$ ):

$$
\forall S \in 2^{N} \backslash\{\emptyset\}, \forall i \in S: x_{S, i}= \begin{cases}r_{i}-\frac{r_{i}}{r(S)} c(S) & \text { if } v(S)>0, \\ 0 & \text { if } v(S)=0 .\end{cases}
$$

Clearly, $\sum_{i \in S} x_{S, i}=v(S)$ for each $S \in 2^{N} \backslash\{\emptyset\}$. Moreover, let $S, T \in 2^{N} \backslash\{\emptyset\}, i \in N$, and assume that $i \in S \subseteq T$. If $v(S)=0$, then $x_{S, i}=0 \leq x_{T, i}$. So assume $v(S)>0$. Then $v(S)=r(S)-c(S)$ and by monotonicity, also $v(T)=r(T)-c(T)>0$. Let $i \in S$. The inequalities $r(S) \leq r(T), c(S) \geq c(T)>0$, and $r_{i} \geq 0$ imply that

$$
x_{S, i}=r_{i}\left(1-\frac{c(S)}{r(S)}\right) \leq r_{i}\left(1-\frac{c(T)}{r(T)}\right)=x_{T, i} .
$$

Hence, $\left(x_{S, i}\right)_{S \subseteq N, i \in S}$ with $x_{S, i}$ defined as in (2) defines a PMAS for the shortest path game $(N, v)$. These conclusions are summarized in the following proposition.

Proposition 3.2 Let $\left\langle N, V,\left(A_{i}\right)_{i \in N}, w,\left(r_{i}\right)_{i \in N}\right\rangle$ be a shortest path problem and $(N, v)$ the associated shortest path game. Then $(N, v)$ is totally balanced and has a populationmonotonic allocation scheme.

The result that the collection

$$
B=\left\{x \in \mathbb{R}^{N} \mid x(N)=v(N) \text { and } x_{i} \in\left[0, r_{i}\right] \text { for each } i \in N\right\}
$$

yields a nonempty subset of the core is very appealing from a computational point of view. To compute core elements, it is not necessary to compute the value $v(S)$ of each coalition $S \in 2^{N} \backslash\{\emptyset\}$. It suffices to determine $v(N)=r(N)-c(N)$, i.e, one only needs to compute the costs of a single shortest path instead of computing a shortest path for every coalition.

Finding elements of the set $B$ comes down to dividing the $\operatorname{costs} c(N)$ of a shortest path over the involved players. Each player $i \in N$ can take a share between zero and his reward $r_{i}$. This is exactly a taxation problem (or - under a slightly different interpretation a bankruptcy problem) where the amount to be paid equals $c(N)$ and each player $i \in N$ has a taxable income $r_{i}$. The numerous allocation rules that have been proposed in the literature on taxation problems (cf. Young, 1987, 1990) and bankruptcy problems (cf. Aumann and Maschler, 1985) therefore yield core elements of the shortest path game. 


\section{A rule favoring s-veto players}

In the previous section nonemptiness of the core of shortest path games was established by indicating that every efficient allocation in which all players receive a nonnegative amount not exceeding their reward yields a core element. There may be players, however, who are of such importance to the generation of a shortest path, that they can claim in the core a payoff that is higher than their reward. In this section, we show that these players are exactly the players owning an arc of every shortest path. Fragnelli et al. (1999) refer to these players as shortest veto players or s-veto players. In their paper, s-veto players must exist to guarantee nonemptiness of the core.

We formulate an allocation rule that indeed favors such s-veto players and still yields a core allocation.

Definition 4.1 Let $\left\langle N, V,\left(A_{i}\right)_{i \in N}, w,\left(r_{i}\right)_{i \in N}\right\rangle$ be a shortest path problem and $(N, v)$ the associated shortest path game. A player $i \in N$ is a shortest veto player or s-veto player if player $i$ owns at least one arc on every shortest path. The (possibly empty) set of s-veto players is denoted by $T \subseteq N$.

In the core of a shortest path game, only s-veto players can receive a payoff strictly exceeding their reward.

Proposition 4.2 Let $\left\langle N, V,\left(A_{i}\right)_{i \in N}, w,\left(r_{i}\right)_{i \in N}\right\rangle$ be a shortest path problem and $(N, v)$ the associated shortest path game. Let $x \in C(N, v)$ and $i \in N$. If $x_{i}>r_{i}$, then $i \in T$.

Proof. Suppose that $i \notin T$. Then there exists a shortest path not using any of $i$ 's arcs: $c(N)=c(N \backslash\{i\})$. Therefore, the following holds:

$$
\begin{aligned}
x(N) & =v(N) \\
& =r(N)-c(N) \\
& =r_{i}+r(N \backslash\{i\})-c(N \backslash\{i\}) \\
& \leq r_{i}+v(N \backslash\{i\}) \\
& <x_{i}+v(N \backslash\{i\}) .
\end{aligned}
$$

This implies that $x(N \backslash\{i\})<v(N \backslash\{i\})$, contradicting $x \in C(N, v)$. Thus, $i$ has to be an s-veto player.

Without s-veto players, the shortest path cannot be realized. The rule we propose is based on a consideration of opportunity costs, i.e, the costs of the next to shortest path. The s-veto players are rewarded for their powerful position in the network, whereas the remaining players are required to make a large contribution to the costs.

This idea is clearly not applicable if either the set of s-veto players or its complement is empty, or if there is no next to shortest path (i.e., all paths from source to sink have 
the same length). In those cases, the costs $c(N)$ of the shortest path are simply divided proportional to the rewards of the players:

$$
\forall i \in N: x_{i}=r_{i}-\frac{r_{i}}{r(N)} c(N)
$$

Assume now that there are both s-veto players $(T \neq \emptyset)$ and non-s-veto players $(N \backslash T \neq \emptyset)$ and that not all paths have the same length. For notational convenience, let $c_{1}$ denote the costs $c(N)$ of the shortest path, i.e., of the first-best alternative, and let $c_{2}$ denote the costs of the next to shortest path. Formally, if $P$ denotes the set of all paths:

$$
c_{2}=\min _{p \in P: \cos t(p)>c_{1}} \operatorname{cost}(p)
$$

By definition:

$$
c_{1}<c_{2}
$$

The non-s-veto players in $N \backslash T$ pay a share of the costs of the second-best path proportional to their reward (or their entire reward, if their funds are insufficient to cover the $\left.\operatorname{costs} c_{2}\right)$ :

$$
\forall i \in N \backslash T \quad: \quad x_{i}=r_{i}-\frac{r_{i}}{r(N \backslash T)} c_{2} \wedge r(N \backslash T) .
$$

This indicates that the players that do not own arcs on every shortest path are burdened to a high extent: together they contribute an amount equal to $c_{2} \wedge r(N \backslash T)$. There are two possibilities. First of all, it is possible that this amount suffices to pay for the shortest path: $c_{2} \wedge r(N \backslash T)>c_{1}$. In that case, there is an excess contribution of $c_{2} \wedge r(N \backslash T)-c_{1}$. This excess is split over the s-veto players in proportion to their rewards. Secondly, it is possible that the amount contributed by the non-s-veto players does not exceed the costs of the shortest path: $c_{2} \wedge r(N \backslash T) \leq c_{1}$. In that case, the remaining costs $c_{1}-c_{2} \wedge r(N \backslash T)$ are contributed by the s-veto players in proportion to their rewards. These two cases give rise to the following payoff to s-veto players:

$$
\forall i \in T \quad: \quad x_{i}=r_{i}+\frac{r_{i}}{r(T)}\left[c_{2} \wedge r(N \backslash T)-c_{1}\right]
$$

Example 4.3 In the shortest path game of Examples 2.2 and 2.4 the unique shortest path goes via the vertex $v$ and is owned by players 1 and 2 . These are the s-veto players: $T=\{1,2\}, N \backslash T=\{3\}$. The shortest path has costs $c_{1}=2$, whereas the second-best alternative has costs $c_{2}=3$. According to (5), player 3 is charged to pay the costs $c_{2}$, which is possible, since his reward equals $8: x_{3}=8-3=5$. The non-s-veto player 3 has already contributed an amount of 3 to cover the costs $c_{2}$. The costs of the shortest path are only $c_{1}=2$. The excess of $3-2=1$ is split over the s-veto players in proportion to their rewards: $x_{1}=x_{2}=8+\frac{1}{2} * 1=8 \frac{1}{2}$. Notice that this allocation $x=\left(8 \frac{1}{2}, 8 \frac{1}{2}, 5\right)$ is a core allocation.

Theorem 4.4 The allocation rule defined by (5) and (6) - or by (3) in case the set of s-veto players or its complement is empty, or if all paths have the same length - yields a core element. 
Proof. If the allocation rule is defined by proportional sharing (3), this yields a core allocation, as was shown in Section 3. It remains to consider the case in which the rule is determined by expressions (5) and (6). Notice:

(a) $x(N)=r(N)-c_{1}=r(N)-c(N)=v(N)$, so the allocation is efficient.

(b) $\forall i \in N \backslash T: x_{i}=r_{i}\left(1-\frac{c_{2} \wedge r(N \backslash T)}{r(N \backslash T)}\right) \geq 0$.

(c) $\forall i \in T$ :

- if $c_{2} \wedge r(N \backslash T)-c_{1} \geq 0$, then $x_{i}=r_{i}\left(1+\frac{c_{2} \wedge r(N \backslash T)-c_{1}}{r(T)}\right) \geq 0$.

- if $c_{2} \wedge r(N \backslash T)-c_{1}<0$, then $c_{2} \wedge r(N \backslash T)=r(N \backslash T)$ by (4), so $x_{i}=$ $r_{i}\left(1+\frac{r(N \backslash T)-c_{1}}{r(T)}\right) \geq 0$, since $r(N)-c(N)=r(N)-c_{1} \geq 0$ implies that $1+$ $\frac{r(N \backslash T)-c_{1}}{r(T)} \geq 0$.

Let $S \in 2^{N} \backslash\{\emptyset\}$. To show: $x(S) \geq v(S)$. By (b) and (c) above, this is true if $v(S)=0$, so assume $v(S)=r(S)-c(S)>0$. Discern three cases.

Case 1: $r(N \backslash T) \leq c_{1}$.

Then (4) implies $c_{2} \wedge r(N \backslash T)=r(N \backslash T) \leq c_{1}$. So by (5), (6), and (b) and (c) above: $x_{i} \in\left[0, r_{i}\right]$ for each player $i \in N$. Together with $x(N)=v(N)$ and Proposition 3.1, it follows that $x \in C(N, v)$.

Case 2: $r(N \backslash T)>c_{1}$ and $c(S)=c_{1}$.

Since $c(S)=c_{1}$, it follows that $S \cap T=T$. Hence:

$$
\begin{aligned}
x(S) & =r(S \cap(N \backslash T))-\frac{r(S \cap(N \backslash T))}{r(N \backslash T)} c_{2} \wedge r(N \backslash T) \\
& +r(S \cap T)+\frac{r(S \cap T)}{r(T)}\left(c_{2} \wedge r(N \backslash T)-c_{1}\right) \\
& =r(S)-\frac{r(S \cap(N \backslash T))}{r(N \backslash T)} c_{2} \wedge r(N \backslash T)+c_{2} \wedge r(N \backslash T)-c_{1} \\
& =r(S)+\left(1-\frac{r(S \cap(N \backslash T))}{r(N \backslash T)}\right) c_{2} \wedge r(N \backslash T)-c_{1} \\
\geq & r(S)-c_{1} \\
& =r(S)-c(S) \\
& =v(S) .
\end{aligned}
$$

Case 3: $r(N \backslash T)>c_{1}$ and $c(S)>c_{1}$. 
Since $c(S)>c_{1}$ and $c_{2}$ are the costs of the second-best alternative, it follows that $c(S) \geq c_{2}$. Moreover, $c_{2} \wedge r(N \backslash T)-c_{1} \geq 0$. Hence:

$$
\begin{aligned}
x(S) & =r(S \cap(N \backslash T))-\frac{r(S \cap(N \backslash T))}{r(N \backslash T)} c_{2} \wedge r(N \backslash T) \\
& +r(S \cap T)+\frac{r(S \cap T)}{r(T)}\left(c_{2} \wedge r(N \backslash T)-c_{1}\right) \\
& =r(S)-\frac{r(S \cap(N \backslash T))}{r(N \backslash T)} c_{2} \wedge r(N \backslash T)+\frac{r(S \cap T)}{r(T)}\left(c_{2} \wedge r(N \backslash T)-c_{1}\right) \\
& \geq r(S)-\frac{r(S \cap(N \backslash T))}{r(N \backslash T)} c_{2} \wedge r(N \backslash T) \\
& \geq r(S)-1 * c(S) \\
& =v(S) .
\end{aligned}
$$

This finishes the proof.

Since the players in $N \backslash T$ are charged to pay the opportunity costs (or to contribute their entire reward if they have insufficient funds to pay the opportunity costs), one might ask what happens if the opportunity costs are defined to be $c(N \backslash T)$, the costs of a shortest path owned by coalition $N \backslash T$, instead of $c_{2}$. This, however, does not necessarily yield a core allocation.

Example 4.5 Suppose that $c_{2}$ in (5) and (6) is replaced by $c(N \backslash T)$. In the shortest path game of Example 2.2 and 2.4, $c(N \backslash T)=c(\{3\})=6$. If player 3 is charged to pay these costs and the excess $6-c_{1}=6-2=4$ is split over the s-veto players in proportion to their rewards, one obtains the allocation $y=(10,10,2)$, which is not a core allocation, since $y_{1}+y_{3}=12<v(\{1,3\})=13$.

\section{Noncooperative support for the core}

In this final section, we introduce a noncooperative game associated with a shortest path problem $\left\langle N, V,\left(A_{i}\right)_{i \in N}, w,\left(r_{i}\right)_{i \in N}\right\rangle$ and prove that the core allocations of the cooperative shortest path game coincide with payoffs to strong Nash equilibria in the noncooperative game.

The strategic game $\left\langle N,\left(X_{i}\right)_{i \in N},\left(u_{i}\right)_{i \in N}\right\rangle$ is defined as follows: The player set is $N$, the set of players involved in the shortest path problem. A strategy of player $i \in N$ is a pair $s_{i}=\left(p_{i}, m_{i}\right)$ consisting of

- a path $p_{i}$ from source to sink;

- an amount of money $m_{i} \in \mathbb{R}$, indicating how much player $i$ is willing to contribute to the costs of his chosen path $p_{i}$. In principle $m_{i} \geq 0$, but if player $i$ owns arcs on 
the path $p_{i}$, he is also allowed to make claims rather than contributions, i.e, then he can also contribute $m_{i}<0$.

The set of strategies of player $i \in N$ is denoted by $X_{i}$. Write $X=\prod_{i \in N} X_{i}$ and $s=$ $\left(p_{i}, m_{i}\right)_{i \in N} \in X$. Given a strategy profile $s=\left(p_{i}, m_{i}\right)_{i \in N} \in X$, a path $p$ is feasible if every arc on this path is owned by a player from the set $\left\{i \in N \mid p_{i}=p\right\}$ choosing this path and if the net contribution generated by these players suffices to pay the costs of the path $p:$

$$
\sum_{i \in N: p_{i}=p} m_{i} \geq \operatorname{cost}(p)
$$

Let $F(s)$ denote the collection of feasible paths given $s \in X$, and define $\delta: X \rightarrow\{0,1\}$ by taking for each $s \in X$ :

$$
\delta(s)= \begin{cases}1 & \text { if } F(s) \neq \emptyset \\ 0 & \text { otherwise. }\end{cases}
$$

The payoffs are such that a player receives his reward if there is a feasible path (see also Remark 5.2 at the end of this section). Contributions are always accepted, whereas a claim (negative contribution) of player $i \in N$ is granted only if the path he chose is feasible. Formally:

$$
\forall i \in N, \forall s \in X: u_{i}(s)= \begin{cases}r_{i} \delta(s)-m_{i} & \text { if } m_{i} \geq 0 \text { or if } m_{i}<0 \text { and } p_{i} \in F(s), \\ r_{i} \delta(s) & \text { if } m_{i}<0 \text { and } p_{i} \notin F(s) .\end{cases}
$$

Recall that a strategy profile $s \in X$ of a game $\left\langle N,\left(X_{i}\right)_{i \in N},\left(u_{i}\right)_{i \in N}\right\rangle$ is a strong $N a s h$ equilibrium if there is no coalition of players that can profitably deviate from $s$, i.e., if there is no coalition $S \in 2^{N} \backslash\{\emptyset\}$ and a strategy combination $\bar{s} \in X$ with $\bar{s}_{i}=s_{i}$ for all $i \in N \backslash S$ such that $u_{i}(\bar{s}) \geq u_{i}(s)$ for each $i \in S$ with strict inequality for at least one player $i \in S$. Denote the collection of strong Nash equilibria of a strategic game $G=\left\langle N,\left(X_{i}\right)_{i \in N},\left(u_{i}\right)_{i \in N}\right\rangle$ by $S N E(G)$. Core elements of the cooperative shortest path game exactly coincide with payoffs to strong Nash equilibria in the strategic shortest path game.

Theorem 5.1 Let $\left\langle N,\left(A_{i}\right)_{i \in N}, w,\left(r_{i}\right)_{i \in N}\right\rangle$ be a shortest path problem, $(N, v)$ the associated cooperative game, and $G=\left\langle N,\left(X_{i}\right)_{i \in N},\left(u_{i}\right)_{i \in N}\right\rangle$ the associated strategic game. Then

$$
C(N, v)=\left\{\left(u_{i}(s)\right)_{i \in N} \mid s \in S N E(G)\right\} .
$$

Proof. To prove that $C(N, v) \subseteq\left\{\left(u_{i}(s)\right)_{i \in N} \mid s \in S N E(G)\right\}$, let $x \in C(N, v)$. Let $p$ be a shortest path owned by the grand coalition $N$. Define the strategy combination $s=\left(p_{i}, m_{i}\right)_{i \in N} \in X$ by setting

$$
\forall i \in N: \quad p_{i}=p \text { and } m_{i}=r_{i}-x_{i}
$$

By Proposition 4.2, only s-veto players can have $x_{i}>r_{i}$ and consequently $m_{i}<0$. Since s-veto players indeed own arcs on the shortest path $p$, the strategy combination $s$ is well-defined: only players owning arcs on $p$ can make claims. Clearly $F(s)=\{p\}$ and 
$u_{i}(s)=r_{i}-m_{i}=x_{i}$ for each $i \in N$. To show that $s \in S N E(G)$, suppose, to the contrary, that coalition $S \in 2^{N} \backslash\{\emptyset\}$ can profitably deviate to strategies $\left(\bar{p}_{i}, \bar{m}_{i}\right)_{i \in S}$. Call the new strategy profile $\bar{s}$ :

$$
\forall i \in N: \bar{s}_{i}= \begin{cases}\left(p_{i}, m_{i}\right) & \text { if } i \in N \backslash S, \\ \left(\bar{p}_{i}, \bar{m}_{i}\right) & \text { if } i \in S .\end{cases}
$$

Since $\bar{s}$ involves a profitable deviation of coalition $S$ :

$$
\begin{array}{ll}
\forall i \in S: & u_{i}(\bar{s}) \geq u_{i}(s), \\
\exists i \in S: & u_{i}(\bar{s})>u_{i}(s) .
\end{array}
$$

Suppose $F(\bar{s})=\emptyset$, i.e., there are no feasible paths in the strategy combination $\bar{s}$. Then $u_{i}(\bar{s}) \leq 0 \leq x_{i}=u_{i}(s)$ for each $i \in S$, contradicting (8). Therefore

$$
F(\bar{s}) \neq \emptyset .
$$

Assume in the remainder of the proof that the members of $S$ all choose a feasible path:

$$
\forall i \in S: \bar{p}_{i} \in F(\bar{s})
$$

This involves no loss of generality: if $S$ can profitably deviate at all, then $S$ can in particular profitably deviate to a strategy combination satisfying (10), since players $i \in S$ for which $\bar{p}_{i} \notin F(\bar{s})$ can deviate from $\bar{s}$ by choosing $\tilde{p}_{i} \in F(\bar{s})$, which is possible by (9), and $\widetilde{m}_{i}=0$, without decreasing the payoffs of the members of $S$. Results (9) and (10) imply that $u_{i}(\bar{s})=r_{i}-\bar{m}_{i}$ for each $i \in S$, possibly with $\bar{m}_{i}=0$. With $(7)$ and $(8)$ :

$$
\begin{array}{ll}
\forall i \in S: & u_{i}(\bar{s})=r_{i}-\bar{m}_{i} \geq u_{i}(s)=r_{i}-m_{i}, \\
\exists i \in S: & u_{i}(\bar{s})=r_{i}-\bar{m}_{i}>u_{i}(s)=r_{i}-m_{i} .
\end{array}
$$

Summing over $i \in S$ we find that

$$
\sum_{i \in S} \bar{m}_{i}<\sum_{i \in S} m_{i}
$$

The collection $R:=\left\{\bar{p}_{i} \mid i \in S\right\}$ of paths selected by the members of $S$ in strategy combination $\bar{s}$ is a nonempty, finite set. By (10):

$$
R \subseteq F(\bar{s}) .
$$

To derive a contradiction from our initial assumption that $S$ can profitably deviate from $s$ to $\bar{s}$, we discern two cases:

- Suppose that the shortest path $p$ used to define the initial strategy combination $s$ is chosen by a member of $S$ after deviation to $\bar{s}: p \in R$. This means that in the strategy combination $\bar{s}$ every player $i \in N$ in the strategy combination $\bar{s}$ chooses a path in the set $R$ : members of $S$ do so by definition and players in $N \backslash S$ choose the path $p \in R$. By (14), all paths in $R$ are indeed paid for by the players in $N$ :

$$
\sum_{i \in S} \bar{m}_{i}+\sum_{i \in N \backslash S} m_{i} \geq \sum_{q \in R} \operatorname{cost}(q) \geq \operatorname{cost}(p)=c(N)=\sum_{i \in N} m_{i} .
$$

This implies $\sum_{i \in S} \bar{m}_{i} \geq \sum_{i \in S} m_{i}$, contradicting (13). 
- Suppose $p \notin R$, i.e, $\bar{p}_{i} \neq p$ for each $i \in S$. This means that in the strategy combination $\bar{s}$ all players in $S$ choose a path from the set $R$ and all players in $N \backslash S$ choose the path $p \notin R$. By (14), all paths in $R$ are indeed paid for by the players in $S$ :

$$
\sum_{i \in S} \bar{m}_{i} \geq \sum_{q \in R} \operatorname{cost}(q) \geq c(S)
$$

where the second inequality follows from the fact that all paths in $R \subseteq F(\bar{s})$ are owned by the members of $S$ and therefore cost at least as much as the shortest path owned by coalition $S$. Since $\left(u_{i}(s)\right)_{i \in N}=x \in C(N, v)$, we also have, using (11) and (12), that

$$
r(S)-\sum_{i \in S} \bar{m}_{i}=\sum_{i \in S} u_{i}(\bar{s})>\sum_{i \in S} u_{i}(s) \geq v(S) \geq r(S)-c(S),
$$

from which we conclude $\sum_{i \in S} \bar{m}_{i}<c(S)$, contradicting (15).

Since both cases yield a contradiction, it must be that $s \in S N E(G)$, as was to be shown.

To prove that $C(N, v) \supseteq\left\{\left(u_{i}(s)\right)_{i \in N} \mid s \in S N E(G)\right\}$, let $s=\left(p_{i}, m_{i}\right)_{i \in N} \in S N E(G)$. Each player $i \in N$ with $p_{i} \notin F(s)$ can avoid a negative payoff by making a zero contribution (or a negative contribution if he owns arcs on his selected path $p_{i}$ ). Therefore

$$
\forall i \in N: \quad u_{i}(s) \geq 0
$$

$$
\forall i \in N: \text { if } p_{i} \notin F(s) \text {, then } m_{i} \leq 0 \text { and } u_{i}(s)=r_{i} \delta(s) \text {. }
$$

If $F(s)=\emptyset$, then (17) implies that $u_{i}(s)=0$ for all $i \in N$. But then $s$ is not a strong Nash equilibrium, since the previous part of the proof indicates that the grand coalition $N$ can deviate to a strategy combination yielding a payoff $x \in C(N, v)$. This is a profitable deviation, since individual rationality implies that $x_{i} \geq v(\{i\}) \geq 0$ for each $i \in N$ and by efficiency $\sum_{i \in N} x_{i}=v(N)>0$, where the inequality follows from assumption (1), so at least one player $i \in N$ obtains a strictly positive payoff. As a consequence, $F(s) \neq \emptyset$.

In fact, $F(s)$ must be a singleton set. Suppose, to the contrary, that $F(s)$ consists of at least two elements. Let $p \in F(s)$ and $S=\left\{i \in N \mid p_{i}=p\right.$ and $\left.m_{i}>0\right\}$. Coalition $S$ is nonempty, since $p \in F(s)$ implies that $\sum_{i \in N: p_{i}=p} m_{i} \geq \operatorname{cost}(p)>0$. Each player $i \in S$ receives payoff $u_{i}(s)=r_{i}-m_{i}$. However, deviating to $\left(\bar{p}_{i}, \bar{m}_{i}\right)_{i \in S}$ with $\bar{p}_{i}=p$ and $\bar{m}_{i}=0$ would yield a set of feasible paths $F(s) \backslash\{p\}$, which is nonempty by our assumption that $F(s)$ contains at least two elements. Hence, each member $i \in S$ after deviating receives a payoff $r_{i}>r_{i}-m_{i}=u_{i}(s)$, which is an improvement, contradicting $s \in S N E(G)$. Therefore, there exists a unique path $p$ such that

$$
F(s)=\{p\} .
$$

Combining (17) and (18) yields

$$
\forall i \in N: u_{i}(s)= \begin{cases}r_{i}-m_{i} & \text { if } p_{i}=p \\ r_{i} & \text { if } p_{i} \neq p .\end{cases}
$$


The path $p$ has to be a shortest path. Suppose, to the contrary, that $p$ is not a shortest path. Select a shortest path $q$. Since $F(s)=\{p\}$ :

$$
\sum_{i \in N: p_{i}=p} m_{i}=\sum_{i \in N: p_{i}=p \text { and } m_{i} \leq 0} m_{i}+\sum_{i \in N: p_{i}=p \text { and } m_{i}>0} m_{i} \geq \operatorname{cost}(p)>\operatorname{cost}(q) .
$$

This implies the existence of a $\lambda \in(0,1)$ such that

$$
\sum_{i \in N: p_{i}=p \text { and } m_{i} \leq 0} m_{i}+\sum_{i \in N: p_{i}=p \text { and } m_{i}>0} \lambda m_{i}=\operatorname{cost}(q) .
$$

Let coalition $S=\left\{i \in N \mid p_{i}=p\right\}$ deviate to $\left(\bar{p}_{i}, \bar{m}_{i}\right)_{i \in S}$ with

$$
\forall i \in S: \quad \bar{p}_{i}=q \text { and } \bar{m}_{i}= \begin{cases}m_{i} & \text { if } m_{i} \leq 0, \\ \lambda m_{i} & \text { if } m_{i}>0 .\end{cases}
$$

Let $\bar{s}$ denote the strategy combination after this deviation of coalition $S$. By $(20): F(\bar{s})=$ $\{q\}$. Consequently

$$
\forall i \in S: u_{i}(\bar{s})= \begin{cases}r_{i}-m_{i}=u_{i}(s) & \text { if } m_{i} \leq 0, \\ r_{i}-\lambda m_{i}>r_{i}-m_{i}=u_{i}(s) & \text { if } m_{i}>0 .\end{cases}
$$

This is a profitable deviation by coalition $S$, contradicting $s \in S N E(G)$. Conclude that $p$ in (18) is indeed a shortest path.

From (18) it follows that $\sum_{i \in N: p_{i}=p} m_{i} \geq \operatorname{cost}(p)$. Analogous to the proof in the previous paragraph that $p$ is a shortest path, one can show that the weak inequality must be an equality:

$$
\sum_{i \in N: p_{i}=p} m_{i}=\operatorname{cost}(p)=c(N)
$$

(Otherwise a profitable deviation exists by letting the contributors $\left\{i \in N \mid p_{i}=\right.$ $p$ and $\left.m_{i}>0\right\}$ pay slightly less, but sufficient to cover the costs of the shortest path p.) Combining (19) and (21) yields

$$
\sum_{i \in N} u_{i}(s)=\sum_{i \in N: p_{i}=p}\left(r_{i}-m_{i}\right)+\sum_{i \in N: p_{i} \neq p} r_{i}=r(N)-\sum_{i \in N: p_{i}=p} m_{i}=r(N)-c(N)=v(N) .
$$

To show that $\left(u_{i}(s)\right)_{i \in N} \in C(N, v)$, it remains to show that $\sum_{i \in S} u_{i}(s) \geq v(S)$ for each coalition $S \in 2^{N} \backslash\{\emptyset, N\}$. Suppose, to the contrary, that $\sum_{i \in S} u_{i}(s)<v(S)$ for coalition $S \in 2^{N} \backslash\{\emptyset, N\}$. By $(16): v(S)=r(S)-c(S)>0$. By $(19)$ :

$$
\sum_{i \in S} u_{i}(s)=r(S)-\sum_{i \in S: p_{i}=p} m_{i}<v(S)=r(S)-c(S)
$$

which implies that the net contribution $\sum_{i \in S: p_{i}=p} m_{i}$ of players in $W=\left\{i \in S: p_{i}=p\right\}$ exceeds $c(S)>0$ :

$$
\sum_{i \in S: p_{i}=p} m_{i}>c(S)
$$

Therefore $W \neq \emptyset$. Discern two cases: 
- if $c(S)>c(N)$, then $S$ owns a path $q \neq p$ with $\operatorname{cost}(q)=c(S)$;

- if $c(S)=c(N)$, then $\sum_{i \in S: p_{i}=p} m_{i}>c(N)$ by (22). Combining this with (21) yields $\sum_{i \in N \backslash S: p_{i}=p} m_{i}<0$. Consequently, $m_{i}<0$ for some player $i \in N \backslash S$ with $p_{i}=p$. By definition of a strategy, this player $i \in N \backslash S$ owns at least one arc on the path $p$, otherwise he is not allowed to make negative contributions. This implies that $S$ does not own all arcs on the path $p$. But $c(S)=c(N)$ implies that a path $q \neq p$ exists that is owned by $S$ and satisfies $\operatorname{cost}(q)=c(S)$.

In both cases $S$ owns a path $q \neq p$ with $\operatorname{cost}(q)=c(S)$. By $(22)$ there exists a $\lambda \in(0,1)$ such that

$$
\sum_{i \in S: p_{i}=p \text { and } m_{i} \leq 0} m_{i}+\sum_{i \in S: p_{i}=p \text { and } m_{i}>0} \lambda m_{i}=c(S)=\operatorname{cost}(q) .
$$

Let $S$ deviate to strategies $\left(\bar{p}_{i}, \bar{m}_{i}\right)_{i \in S}$ with

$$
\forall i \in S: \bar{p}=q \text { and } \bar{m}_{i}= \begin{cases}0 & \text { if } p_{i} \neq p \\ m_{i} & \text { if } p_{i}=p \text { and } m_{i} \leq 0 \\ \lambda m_{i} & \text { if } p_{i}=p \text { and } m_{i}>0\end{cases}
$$

Let $\bar{s}$ denote the strategy combination after this deviation of coalition $S$. By $(23): q \in$ $F(\bar{s})$. Consequently

$$
\forall i \in S: \quad u_{i}(\bar{s})= \begin{cases}r_{i}=u_{i}(s) & \text { if } p_{i} \neq p, \\ r_{i}-m_{i}=u_{i}(s) & \text { if } p_{i}=p \text { and } m_{i} \leq 0, \\ r_{i}-\lambda m_{i}>r_{i}-m_{i}=u_{i}(s) & \text { if } p_{i}=p \text { and } m_{i}>0\end{cases}
$$

This is a profitable deviation by coalition $S$, contradicting $s \in S N E(G)$. Conclude that indeed $\sum_{i \in S} u_{i}(s) \geq v(S)$ for each $S \in 2^{N} \backslash\{\emptyset, N\}$, as was to be shown.

Remark 5.2 In the strategic shortest path game, a player receives his reward if the set of feasible paths is nonempty, irrespective of whether he chooses a feasible path as part of his strategy. An obvious modification of the strategic game would be to give a player his reward only if he chooses a feasible path. One can modify the proof to show that also in that case the conclusion of Theorem 5.1 holds.

An additional modification would be to assign payoff zero to players selecting a nonfeasible path: players that contribute to a nonfeasible path are paid back their contribution, whereas players making claims on an infeasible path are denied their claim. The resulting payoff functions $\left(u_{i}\right)_{i \in N}$ would be defined as follows:

$$
\forall i \in N, \forall s \in X: u_{i}(s)= \begin{cases}r_{i}-m_{i} & \text { if } p_{i} \in F(s), \\ 0 & \text { if } p_{i} \notin F(s) .\end{cases}
$$

Also under this modification, Theorem 5.1 remains valid.

Both alternative definitions of the payoff functions require only minor changes in the proof of Theorem 5.1. The proof, however, would become longer, without giving more insight. We therefore did not adopt these alternative definitions. 
By definition of a strategy, players are allowed to make claims only on those paths on which they own arcs. If players would have been allowed to make claims on paths where they own no arcs, the conclusion of Theorem 5.1 is no longer valid. Consider, for instance, the shortest path problem in which $N=\{1,2\}, r_{1}=r_{2}=3$, and the network is given in Figure 2. Suppose we also allow player 2 to make claims on the unique path. If both

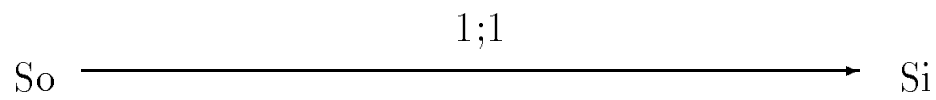

Figure 2: Core and strong Nash equilibria

players select the shortest path, player 1 contributes 2 , and player 2 contributes -1 , then the path is feasible and the players have payoffs $3-2=1$ and $3-(-1)=4$, respectively. It is easy to see that this is a strong Nash equilibrium, but since the non-s-veto player 2 receives a payoff $4>3=r_{2}$, Proposition 4.2 implies that the payoff vector $(1,4)$ is not a core allocation.

\section{References}

Aumann R.J. And Maschler M. (1985): "Game theoretic analysis of a bankruptcy problem from the Talmud", Journal of Economic Theory, 36, 195-213.

CURIEL I. (1997): Cooperative game theory and applications: Cooperative games arising from combinatorial optimization problems, Dordrecht: Kluwer Academic Publishers.

Dijkstra E.W. (1959): “A note on two problems in connection with graphs", $N u^{-}$ merische Mathematik, 1, 269-271.

Fragnelli V., García-Jurado I., and Méndez-Naya L. (1999): “On shortest path games", to appear in Mathematical Methods of Operations Research.

SPRUMONT Y. (1990): "Population monotonic allocation schemes for cooperative games with transferable utility", Games and Economic Behavior, 2, 378-394.

Young H.P. (1990): "Progressive taxation and equal sacrifice", American Economic Review, 80, 253-266.

YOUNG H.P. (1987): "On dividing an amount according to individual claims or liabilities", Mathematics of Operations Research, 12, 398-414. 